\title{
'Stop' that ambulance! New physics at the LHC?
}

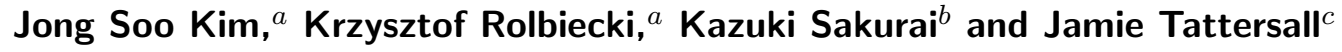 \\ ${ }^{a}$ Instituto de Física Teórica, IFT-UAM/CSIC, \\ C/ Nicolás Cabrera, 13-15, Cantoblanco, 28049 Madrid, Spain \\ ${ }^{b}$ Department of Physics, King's College London, \\ Strand, London, WC2R 2LS, U.K. \\ ${ }^{c}$ University of Heidelberg, Institut für Theoretische Physik, \\ Philosophenweg 16, D-69120 Heidelberg, Germany \\ E-mail: jong.kim@csic.es, krzysztof.rolbiecki@desy.de, \\ kazuki.sakurai@kcl.ac.uk, tattersall@thphys.uni-heidelberg.de
}

ABSTRACT: A number of LHC searches now display intriguing excesses. Most prominently, the measurement of the $W^{+} W^{-}$cross-section has been consistently $\sim 20 \%$ higher than the theoretical prediction across both ATLAS and CMS for both 7 and $8 \mathrm{TeV}$ runs. More recently, supersymmetric searches for final states containing two or three leptons have also seen more events than predicted in certain signal regions. We show that a supersymmetric model containing a light stop, winos and binos can consistently match the data. We perform a fit to all measurements and searches that may be sensitive to our model and find a reduction in the log-likelihood of 15.4 compared to the Standard Model which corresponds to 3.5- $\sigma$ once the extra degrees of freedom in the fit are considered.

KEYWORDS: Supersymmetry Phenomenology

ARXIV EPRINT: 1406.0858 


\section{Contents}

1 Introduction 1

2 Tools and measurements 3

$2.1 W^{+} W^{-}$measurements 4

2.2 SUSY di-lepton search 5

2.3 Leptonic squark and gluino searches 5

$\begin{array}{lll}2.4 & \text { SUSY tri-lepton searches } & 6\end{array}$

2.5 Stop searches 6

$\begin{array}{lll}3 & \text { Scan } & 6\end{array}$

4 Results $\quad 8$

4.1 Di-lepton channels 8

4.2 Tri-lepton channels $\quad 9$

$\begin{array}{ll}4.3 \text { Combined analysis } & 11\end{array}$

5 Discussion $\quad 12$

6 Conclusions $\quad 14$

$\begin{array}{ll}\text { A Signal region events } & 15\end{array}$

\section{Introduction}

After two years of running, the ATLAS and CMS experiments at the Large Hadron Collider (LHC) have performed a large number of Standard Model (SM) measurements and searches of beyond-Standard-Model (BSM) physics. Despite an overall agreement between experimental results and SM predictions, several 2- $\sigma$ excesses can be spotted in some key areas of the data. While one would normally expect some of these measurements to fluctuate around the central values, the picture becomes more intriguing when the excesses point to similar kinematic region and final states. In this paper we re-analyse LHC measurements to test a hypothesis that the overall agreement between the data and theory predictions can be improved by including the production of light supersymmetric [1] top quark partners [2]. This task is performed using two automated computer codes aimed at confronting new physics models with the LhC data, Aтом [3] and Checkmate [4].

A starting point of our analysis are the $W^{+} W^{-}$cross section measurements performed by AtLAs and Cms [5-7]. They show a small excess of $1-2-\sigma$ but remarkably consistent between the two experiments and different centre-of-mass energies $(\mathrm{cms})$. Recent analyses including higher order effects [8-10] increase the discrepancy, calling for more efforts to 
better understand its origin. Apart from a plausible explanation of systematic effects due to e.g. jet veto, one can invoke BSM models where the excess can be explained by the production of supersymmetric (SUSY) particles: charginos [11], stops [2], or sleptons [12].

We would also like to note that the SM measurements of $W^{ \pm}$[13-15], $Z$ [13-16], $W^{ \pm} Z$ [17-19] and $Z Z$ [20-22] production show very good agreement with the theoretical predictions. This hints that a solution cannot be a simple re-normalisation of the crosssection but requires a non-trivial explanation.

More recently, a pair of ATLAS SUSY searches have shown intriguing excesses in soft lepton and missing energy channels that may also support the BSM interpretation. The first, a SUSY search for squarks and gluinos [23] has a $\sim 2.5-\sigma$ excess, in a signal region that looks for a pair of particularly soft muons and missing energy. Secondly, ATLAS has released a tri-lepton search [24] for electroweak states where a number of different signal regions display small excesses: $1.6,1.9$ and $2.1-\sigma$.

In this paper we focus on a simplified model with a light stop, chargino and two neutralinos and ask whether the above excesses can be explained in a consistent way without violating any of the vast number of SUSY searches that have been performed. In particular we concentrate on a model with a stop that is heaviest, a wino-like neutralino and associated charginos that are slightly lighter and a bino-like lightest supersymmetric particle (LSP). We thus find that with stop production the resulting decay chain dominates,

$$
\tilde{t}_{1} \rightarrow \tilde{\chi}_{1}^{ \pm} b \rightarrow \tilde{\chi}_{1}^{0} W^{ \pm(*)} b \rightarrow \tilde{\chi}_{1}^{0} \ell^{ \pm} \nu b
$$

where the $\left.{ }^{*}\right)$ refers to the fact that the $W^{ \pm}$may only be present as an off-shell state if the mass splitting, $m_{\tilde{\chi}_{1}^{ \pm}}-m_{\tilde{\chi}_{1}^{0}}<m_{W^{ \pm}}$.

The stop pair production, $p p \rightarrow \tilde{t}_{1} \tilde{t}_{1}^{*}$, can therefore significantly contribute to the final state used for $W^{+} W^{-}$cross section measurements, two opposite sign leptons and missing transverse energy. We choose small mass difference between the stop and chargino, $m_{\tilde{t}_{1}}-m_{\tilde{\chi}_{1}^{ \pm}}=7 \mathrm{GeV}$, to help to avoid the jet veto used in $W^{+} W^{-}$studies. In addition, the small mass splitting only produces soft $b$-jets and thus makes the model less strongly constrained by direct stop searches. The final state also contributes in e.g. the signal regions of SUSY searches in the di-lepton channel [23, 25] or control regions of Higgs boson measurements [26, 27].

Along with the stop and chargino, the model also contains two neutralinos that may have significant cross sections. Therefore the additional production processes, $p p \rightarrow \tilde{\chi}_{1}^{ \pm} \tilde{\chi}_{2}^{0}$ and $p p \rightarrow \tilde{\chi}_{1}^{+} \tilde{\chi}_{1}^{-}$, should also be taken into account. In particular, contributions form the first process may become visible in the SUSY tri-lepton search [24] mentioned above with the following decays,

$$
\begin{aligned}
\tilde{\chi}_{1}^{ \pm} & \rightarrow \tilde{\chi}_{1}^{0} W^{ \pm(*)} \rightarrow \tilde{\chi}_{1}^{0} \ell^{ \pm} \nu, \\
\tilde{\chi}_{2}^{0} & \rightarrow \tilde{\chi}_{1}^{0} Z^{(*)} \rightarrow \tilde{\chi}_{1}^{0} \ell^{+} \ell^{-} .
\end{aligned}
$$

Therefore, the present study significantly enhances the scope of the previous analysis [2] that focused purely on the $W^{+} W^{-}$cross-section measurement. 
Of course it is important to not only include measurements that display excesses and bias the fit for new physics. Therefore we include all current ATLAS searches that are sensitive to the model in question. Particularly important are the searches for stop and bottom squarks [28, 29], squarks and gluinos with soft leptons [23] and electroweak searches [24, 25]. Every signal region in these searches is included in a global fit to provide a fair comparison with the SM. In addition, the regions in our model space that are excluded by these searches are given in the final results.

One should also note that additional constraints may be present in a model with light top quark partners. For example, such models may contain extra charge- or colour-breaking vacua [30] but since we only work with a simplified model this is beyond the scope of the current work.

The paper is organized as follows. In section 2 we introduce tools used in the analysis and briefly discuss included LHC searches. In section 3 we provide details of the simplified model and methodology of the parameter scan. The results are described in section 4 followed by a discussion in section 5. Finally we summarize our findings in section 6 .

\section{Tools and measurements}

The idea of the study is to provide a global fit to the SUSY model in question and compare this to the SM. In order to perform this task we make use of studies implemented in the experimental re-casting tools Aтом [3] and Checkmate [4]. Both tools make use of a fast detector implementation, Rivet [31] for Atom $^{1}$ and Delphes ${ }^{2}$ [32] for Checkmate. Both setups use FASTJET [33] with the anti- $k_{T}$ algorithm [34, 35] for all analyses. Additional tools were used to calculate the kinematical variable $M_{T 2}$ [36-38].

All SUSY events are generated with HeRwiG $++[39,40]$. For stop production, these events are normalised by the NLO+NLL cross section using NLL-FAST [41-44], while for chargino and neutralino production, we use Prospino-2 [45]. For all production processes we use the CTEQ NLO parton distribution functions [46].

The HeRwIG ++ event generator used in this study does not contain initial state radiation (ISR) calculated at the matrix element level but instead relies on the parton shower as an approximation to this radiation. One may worry that if the signal regions require hard ISR jets, we may underestimate the number of events in these regions. However, none of the studies considered actually requires a particularly hard ISR jet for the relevant signal regions. The 'soft di-muon' channel in the ATLAS squark and gluino search [23] requires a jet with $p_{T}>70 \mathrm{GeV}$ but this is still an momentum range where we expect the parton shower to accurately model radiation since it is well below the typical production centre of mass energies for our model (at least $400 \mathrm{GeV}$ ).

The accuracy of the parton shower has been previously studied [47, 48] and it was found that the Herwig ++ parton shower was within the prediction for matrix element radiation as long as the hardest jet $p_{T}<300 \mathrm{GeV}$. Only once we go into the tails of very

\footnotetext{
${ }^{1}$ ATOM includes experimental efficiencies and smearing modifications to RIVET that allow it to perform like a fast detector simulation.

${ }^{2}$ Checkmate includes a detailed re-tuning of Delphes to match the latest AtLAs experimental setup.
} 


\begin{tabular}{|lrrrr|}
\hline Description & $\begin{array}{r}\sqrt{s} \\
{[\mathrm{TeV}]}\end{array}$ & $\begin{array}{r}\text { Luminosity } \\
{\left[\mathrm{fb}^{-1}\right]}\end{array}$ & $\begin{array}{r}\text { Number } \\
\text { of SR }\end{array}$ & Refs. \\
\hline AtLAS $W^{+} W^{-}$ & 7 & 4.6 & 1 & {$[5]$} \\
CMS $W^{+} W^{-}$ & 7 & 4.9 & 1 & {$[7]$} \\
CMS $W^{+} W^{-}$ & 8 & 3.5 & 1 & {$[6]$} \\
AtLAS Higgs & 8 & 20.7 & 2 & {$[27]$} \\
AtLAS Electroweak $(2 \ell)$ & 8 & 20.3 & 13 & {$[25]$} \\
AtLAS $\tilde{q}$ and $\tilde{g}(1-2 \ell)$ & 8 & 20.1 & 19 & {$[23]$} \\
AtLAS $\tilde{q}$ and $\tilde{g}$ razor $(2 \ell)$ & 8 & 20.3 & 6 & {$[53]$} \\
AtLAS Electroweak $(3 \ell)$ & 8 & 20.3 & 20 & {$[24]$} \\
AtLAS $\tilde{t}(1 \ell)$ & 8 & 20.7 & 8 & {$[29]$} \\
AtLAS $\tilde{t}(2 \ell)$ & 8 & 20.3 & 12 & {$[28]$} \\
CMs $W^{ \pm} Z^{0}$ & 8 & 19.6 & 4 & {$[19]$} \\
AtLAS $W^{ \pm} Z^{0}$ & 8 & 13.0 & 4 & {$[18]$} \\
\hline
\end{tabular}

Table 1. List of experimental analyses used in this study along with the centre-of-mass energy, $\sqrt{s}$, luminosity, number of signal regions (SR) and the corresponding references. All signal regions listed above are included in our global model fit.

hard jet radiation does Herwig ++ fail badly. However, the event rate in these tails is too small to contribute to any of the signal regions anyway.

As stated in the introduction we include the current $W^{+} W^{-}$measurements from both AtLAS and CMS along with all ATLAS searches that may be sensitive. The rational behind only choosing AtLas searches is that both Aтоm and Checkmate have been tuned to ATlas but the CMS tunes have not yet been completed. In addition, it appears that the relevant CMS searches ${ }^{3}$ [50-52] are less sensitive to the model we propose here. All the analyses are listed in table 1 and below we introduce the different analysis classes in more detail.

Throughout the study, all SM backgrounds are taken from the respective experimental publication. However, to validate the various analyses we have generated the dominant SM backgrounds and our results match the experimental results very closely and always to better than $10 \%$.

\section{$2.1 W^{+} W^{-}$measurements}

The first analyses that hinted at a possible excess were the $7 \mathrm{TeV} W^{+} W^{-}$measurements from ATLAS [5] and CMS [7] and both are included in our fit as a simple rate measurement. An early $8 \mathrm{TeV}$ measurement with a small dataset was also produced by CMS [6] so we include this as well. Both measurements target di-lepton plus $E_{T}^{\text {miss }}$ final state.

\footnotetext{
${ }^{3}$ While our study was in the last preparation stages a tri-lepton CMS analysis [49] was made public. The study also shows an excess in the data that may be compatible with our model and we hope to include this soon.
} 
Currently, no $8 \mathrm{TeV}$ measurement has yet been provided by ATLAS so we are eager to find out what this result will be. However, we are able to make an early estimate by examining an AtLas Higgs spin study [27] where the Higgs decays to a $W^{+} W^{-}$pair. Here $W^{+} W^{-}$production is the dominant background and therefore the experiment makes a control region measurement which we can use. Unfortunately, an error analysis is not provided for the control region and we therefore assume that the systematic error will be the same as the CMS analysis (6\%). Considering that the Atras result has $\sim 6$ times as much luminosity and many of the systematic errors are statistically limited we believe that this is a conservative choice.

We furthermore include the Higgs signal region from ref. [27] which differs from the $W^{+} W^{-}$control region solely by the lepton invariant mass cut. Therefore, both regions include the full $W^{+} W^{-}$sample binned in a way that could provide sensitivity to mass difference between chargino and the LSP. The number of expected $W^{+} W^{-}$events in the Higgs signal region of ref. [27] has been renormalized by 1.08 with respect to the Monte Carlo (MC) prediction. ${ }^{4}$ For our purpose we therefore rescale this number, assuming that it was caused by the contamination of $W^{+} W^{-}$control region by the stop production. In this way we obtain a real size of the excess in the observed events with respect to the MC prediction. The precise number of events we assume along with the systematic error and calculated excess are shown in table 2.

\subsection{SUSY di-lepton search}

We include the AtLAS search for chargino and slepton production in the di-lepton channel [25] since the study could provide important limits for stop and gaugino production. It is also interesting to note that like in the Higgs spin study [27], the MC-predicted and observed numbers in the $W^{+} W^{-}$control regions do not match well. This excess can be again neatly explained by the contamination coming from stop production.

As in the Higgs study, the $W^{+} W^{-}$background in the signal regions is found by normalising the Monte Carlo prediction by the excess in the control regions. In this analysis, the rescaling actually leads to a deficit in the number of observed events in the signal regions. We therefore follow the same procedure as for the Higgs study and remove the normalisation on the $W^{+} W^{-}$background.

\subsection{Leptonic squark and gluino searches}

General SUSY searches that include signal regions looking for soft leptons may be particularly relevant for our model. The original motivation for these searches was to look for compressed SUSY or universal extra dimensions (UED) spectra.

The first analysis [23] we consider has both single and di-leptonic signal regions. Of special interest to our model is that a signal region looking for a pair of soft muons displays $\mathrm{a} \sim 2.5-\sigma$ excess (see table 2 ). However, we include all 13 signal regions into our analysis to account for the fact that some of these may also be relevant for our model.

\footnotetext{
${ }^{4}$ Note that even after this rescaling of the $W^{+} W^{-}$background, there is still an excess in the signal region suggesting that the additional contribution falls more often in the low $m_{\ell \ell}$ bin. This feature would promote smaller mass splitting between the chargino and the LSP.
} 
The second analysis [53] is based on the so called 'Razor' variable that re-parametrises the background into a smoothly falling distribution. SUSY is then searched for as a departure from the expected shape. No excess is seen in the analysis and consequently we simply use the search to constrain our model

\subsection{SUSY tri-lepton searches}

Since in our simplified model we have the mass degenerate chargino and neutralino, $\tilde{\chi}_{1}^{ \pm}$ and $\tilde{\chi}_{2}^{0}$, SUSY electroweak searches could also play important role. The most relevant analysis here is the tri-lepton search performed by ATLAS [24]. The study targets final states with three leptons and missing energy. An important feature of this study is the sensitivity to events with low invariant mass of an opposite-sign lepton pairs. Indeed, in several signal regions of low invariant mass one can see the excess of observed events that goes up to 2.1- $\sigma$, see table 2. This results in weaker than expected exclusion limits for chargino-neutralino associated production, notably in the mass region of $\sim 200 \mathrm{GeV}$. Such an excess of events can be easily associated with a neutralino decaying to a pair of leptons provided the mass splitting $m_{\tilde{\chi}_{2}^{0}}-m_{\tilde{\chi}_{1}^{0}}$ is not too large. As we will see later this will be an important contribution to our analysis.

In addition, one may worry that the tri-leptons $\tilde{\chi}_{1}^{ \pm}$and $\tilde{\chi}_{2}^{0}$ decays may enter into the SM $W^{ \pm} Z^{0}$ measurement. For this reason, we also include the analysis of this final state from both AtLas [18] and CMs [19] into our study.

\subsection{Stop searches}

Finally, we include the ATLAS direct searches for light and medium stops with either one [29] or two [28] isolated leptons. Since the mass splitting, $m_{\tilde{t}_{1}}-m_{\tilde{\chi}_{1}^{ \pm}}=7 \mathrm{GeV}$, in our model is small we expect the $b$-quarks produced in this decay to be soft and that these searches will only provide weak constraints. We also note that in the two lepton study a control region measurement of $W^{+} W^{-}$production is made that is then extrapolated to the signal region. We once again remove this normalisation to account for the fact that we expect the control region to be contaminated with signal.

\section{Scan}

For our scan we choose a simplified model with the following mass hierarchy:

$$
m_{\tilde{t}_{1}}>m_{\tilde{\chi}_{1}^{ \pm}}=m_{\tilde{\chi}_{2}^{0}}>m_{\tilde{\chi}_{1}^{0}}
$$

and set the mass difference between the stop and chargino to $m_{\tilde{t}_{1}}-m_{\tilde{\chi}_{1}^{ \pm}}=7 \mathrm{GeV} .{ }^{5}$ Assuming that all other SUSY particles are decoupled, the only allowed decays are given by eqs. (1.1)-(1.3) with the final state branching ratios being given by the SM couplings. The models under investigation are then defined by the two remaining masses: $m_{\tilde{t}_{1}}$ and $m_{\tilde{\chi}_{1}^{0}}$. In order to find the set of parameters that provides the best explanation of the existing

\footnotetext{
${ }^{5}$ We also perform a scan with $m_{\tilde{t}_{1}}-m_{\tilde{\chi}_{1}^{ \pm}}=15 \mathrm{GeV}$ and these results are briefly commented on in section 4.3 .
} 
data we perform a scan in these two parameters. The production cross section of $\tilde{\chi}_{1}^{ \pm} \tilde{\chi}_{2}^{0}$ and $\tilde{\chi}_{1}^{+} \tilde{\chi}_{1}^{-}$is set by assuming wino nature of $\tilde{\chi}_{1}^{ \pm}$and $\tilde{\chi}_{2}^{0}$.

In realistic SUSY models, several modifications of the above scenario could be possible. Firstly, instead of wino-like chargino and neutralino, one could have higgsino-like $\tilde{\chi}_{1}^{ \pm}$accompanied by two neutralinos, $\tilde{\chi}_{2}^{0}$ and $\tilde{\chi}_{3}^{0}$. This will not change the decay patterns above and only the cross section for production of $\tilde{\chi}_{1}^{ \pm} \tilde{\chi}_{2,3}^{0}$ would be reduced compared to the pure wino case. On the other hand, if sleptons are light — but still heavier than the chargino - the branching ratios could be significantly affected enhancing the leptonic decay modes when only three-body decays are allowed. Such a modification could be motivated by dark matter constraints, allowing for larger annihilation cross sections, or by the muon anomalous magnetic moment. Finally, right-handed sleptons lighter than the chargino will not affect its decays because of the vanishing coupling between wino and the right-handed sleptons. In case of staus, this would require a very small left-right mixing and small higgsino component in the light chargino. The main advantage of such a modification, if $m_{\tilde{\ell}}-m_{\tilde{\chi}_{1}^{0}} \sim 10 \mathrm{GeV}$, would be dark matter relic density consistent with current measurements for the bino LSP via co-annihilation with sleptons.

To perform the scan, we generate a grid in the $\left(m_{\tilde{t}_{1}}, m_{\tilde{\chi}_{1}^{0}}\right)$ plane with a step size of $5 \mathrm{GeV}$ in the ranges $150 \mathrm{GeV}<m_{\tilde{t}_{1}}<300 \mathrm{GeV}, 60 \mathrm{GeV}<m_{\tilde{\chi}_{1}^{0}}<300 \mathrm{GeV}$. A corresponding grid is generated for chargino and neutralino production. The number of generated events followed by leptonic decays varies across the plane according to the production cross section and expected number of signal events, in order to reduce the impact of MC statistical errors. Since some of the signal regions of the studies under consideration, notably ATLAS-CONF2013-062 [23] and ATLAS-CONF-2013-089 [53], have very small acceptances, up to 2.5 $10^{6}$ events has been generated around the region of best fit.

The fit was performed with the following test statistics. For signal regions (or measurements) with large numbers of expected and observed events $(>20)$ we use the $\chi^{2}$ statistic,

$$
\chi_{i}^{2}=\frac{\left(n_{i}-\mu_{i}\right)^{2}}{\sigma_{i, \text { stat }}^{2}+\sigma_{i, b}^{2}},
$$

where

$$
\mu_{i}=\mu_{i, b}+\mu_{i, s}
$$

Here, $n_{i}$ is the number of observed events, $\mu_{i, b}$ is the expected number of background events, $\mu_{i, s}$ is the expected number of signal events, $\sigma_{i, \text { stat }}$ and $\sigma_{i, b}$ are the statistical and systematic uncertainty on the expected number of background events for each signal region, $i$.

However, for signal regions with small numbers of expected or observed events, the $\chi^{2}$ statistic becomes unreliable and we therefore use the likelihood-ratio test statistic [54],

$$
-2 \ln \lambda_{j}=-2 \ln \left(\frac{\int_{0}^{\infty} d b_{j}^{\prime} \frac{\left(\mu_{j}^{\prime}\right)^{n_{j}} e^{-\mu_{j}^{\prime}}}{n_{j} !} G\left(\mu_{j}, b_{j}^{\prime}\right)}{\frac{\left(n_{j}\right)^{n_{j}} e^{-n_{j}}}{n_{j} !}}\right) .
$$

for each signal region $j$. 
The systematic uncertainty on the background is included by marginalising over this error and is always assumed to be Gaussian. Specifically, we perform an integral where we shift $\mu_{j} \rightarrow \mu_{j}\left(1+f\left(b^{\prime}\right)\right)$, where $f\left(b^{\prime}\right)$ is drawn from a Gaussian, $G$, with standard deviation, $\sigma_{j, b}$.

The overall test statistic is then given by the summation of the small and large event signal regions, ${ }^{6}$

$$
-2 \ln L=\sum_{i=1}^{N_{h}} \chi_{i}^{2}+\sum_{j=1}^{N_{l}}-2 \ln \lambda_{j} .
$$

Here $N_{h}$ refers to the number of high statistic signal regions with both $\mu_{i}, n_{i} \geq 20$ whereas $N_{l}$ refers to the number of low statistic signal regions with both $\mu_{j}, n_{j}<20$.

We make the assumption that all errors are uncorrelated when we perform the fit. Seeing as many of the detector effects, background samples and signal samples are clearly correlated one may worry about how justified the assumption is. Unfortunately, the experiments often do not even provide a detailed breakdown of systematic errors in a single analysis, let alone provide a detailed correlation matrix. Therefore we found that it is impossible to reliably estimate how big the correlations would be within a single analysis let alone the different disjoint analyses we consider here. In the discussion section 5 , we consider how including correlations may effect our final conclusions.

\section{Results}

Considering that current LHC excesses occur in both two and three lepton signal regions, an interesting question is whether our stop model can simultaneously explain both sets of data. For the di-lepton channels, the dominant process is stop pair production followed by the decay chain,

$$
\tilde{t}_{1} \rightarrow \tilde{\chi}_{1}^{ \pm} b \rightarrow \tilde{\chi}_{1}^{0} W^{ \pm(*)} b \rightarrow \tilde{\chi}_{1}^{0} \ell^{ \pm} \nu b .
$$

However, for the tri-lepton signal, the dominant channel is the electroweak production of $p p \rightarrow \tilde{\chi}_{1}^{ \pm} \tilde{\chi}_{2}^{0}$ with the following decays,

$$
\begin{aligned}
\tilde{\chi}_{1}^{ \pm} & \rightarrow \tilde{\chi}_{1}^{0} W^{ \pm(*)} \rightarrow \tilde{\chi}_{1}^{0} \ell^{ \pm} \nu, \\
\tilde{\chi}_{2}^{0} & \rightarrow \tilde{\chi}_{1}^{0} Z^{(*)} \rightarrow \tilde{\chi}_{1}^{0} \ell^{+} \ell^{-} .
\end{aligned}
$$

Thus, we explore the fits to the two and three leptons channels separately before providing a combination.

\subsection{Di-lepton channels}

For the di-lepton fit $^{7}$ we include all experimental analyses listed in table 2 with the exception of tri-lepton search [24] and the $W^{ \pm} Z^{0}$ measurements $[18,19]$ since stop production will produce a negligible number of events with three leptons.

\footnotetext{
${ }^{6}$ Note that the definition of the likelihood means that $-2 \ln \lambda$ is always positive.

${ }^{7}$ We also include the single lepton stop analysis [29] and single lepton squark and gluino analysis [23] here since these searches are most sensitive to stop production.
} 


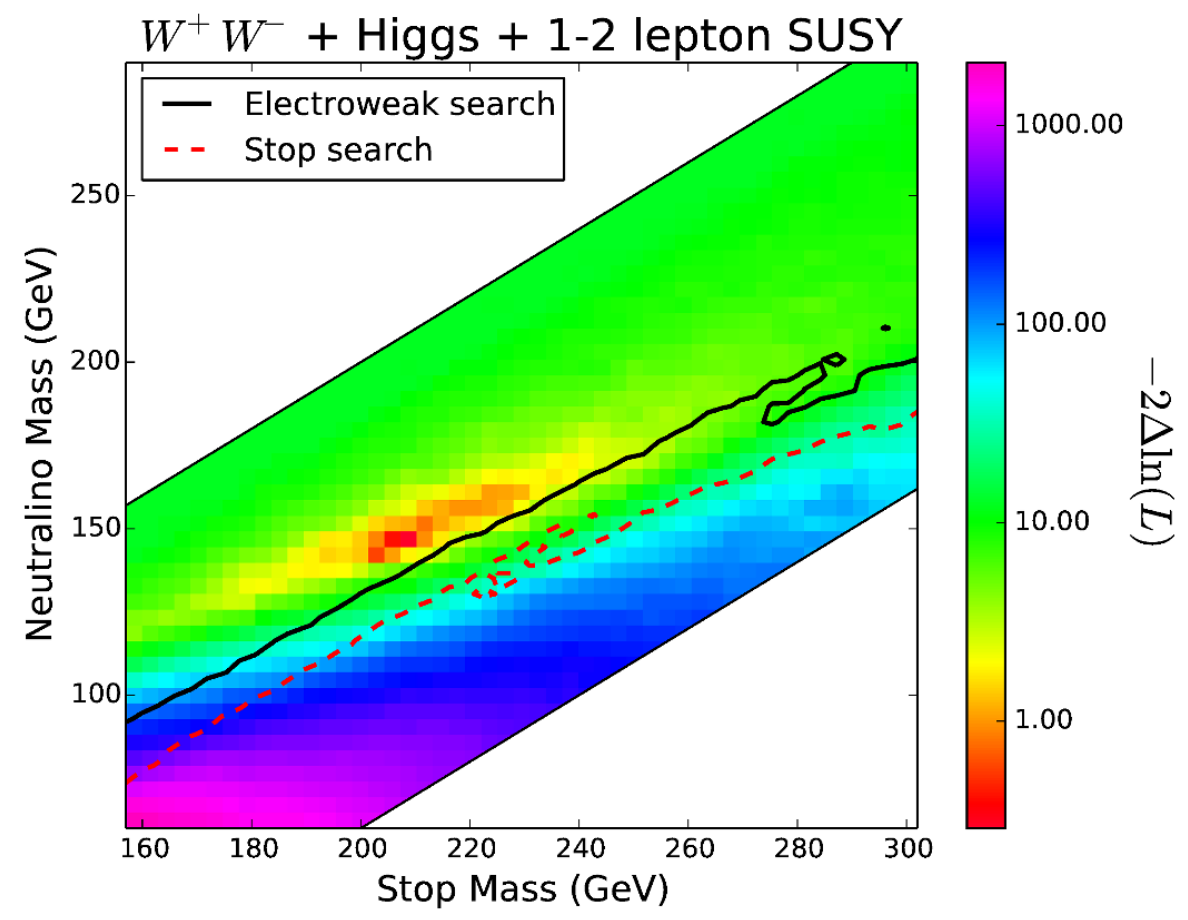

Figure 1. The distribution of $-2 \ln L$ as a function of the masses of the stop, $m_{\tilde{t}_{1}}$, and lightest neutralino, $m_{\tilde{\chi}_{1}^{0}}$. All of the signal regions given in the $W^{+} W^{-}$measurements, any searches with di-lepton final states and the stop searches, table 1 are included in the fit. Also shown are the $95 \%$ CLs exclusion lines given by the dedicated ATLAS search for SUSY electroweak states [25] and the dedicated ATLAS stop search [28].

Performing a scan as a function of $m_{\tilde{t}_{1}}$ and $m_{\tilde{\chi}_{1}^{0}}$ while keeping the mass splitting $m_{\tilde{t}_{1}}-m_{\tilde{\chi}_{1}^{ \pm}}=7 \mathrm{GeV}$ we find that the stop model fits the current data significantly better than the SM as can be seen in figure 1. The best fit point is found to be,

$$
\begin{aligned}
& m_{\tilde{t}_{1}}=207_{-30}^{+40} \mathrm{GeV}, \\
& m_{\tilde{\chi}_{1}^{0}}=145_{-10}^{+25} \mathrm{GeV} .
\end{aligned}
$$

Compared to the SM we find a reduction in the $\log$-likelihood, $-2 \ln L=11.9$, which corresponds to $\sim 3.0-\sigma$ once the extra degrees of freedom in the fit are considered.

In addition we plot the exclusion limits from the most constraining ATLAS supersymmetric searches in figure 1. We find that the electroweak search for two leptons [25] best constrains our model but cannot exclude the best fit point. However, since the exclusion probes mass splittings between the $m_{\tilde{t}_{1}}$ and $m_{\tilde{\chi}_{1}^{0}}$ only $\sim 15 \mathrm{GeV}$ larger than our preferred region, we believe that there is a good chance that these searches can probe the model in the near future.

\subsection{Tri-lepton channels}

For the tri-lepton fit include the dedicated ATLAs search [24] and the $W^{ \pm} Z^{0}$ measurements to concentrate on the electroweak production of $\tilde{\chi}_{1}^{ \pm} \tilde{\chi}_{2}^{0}$ followed by the decay chains given 


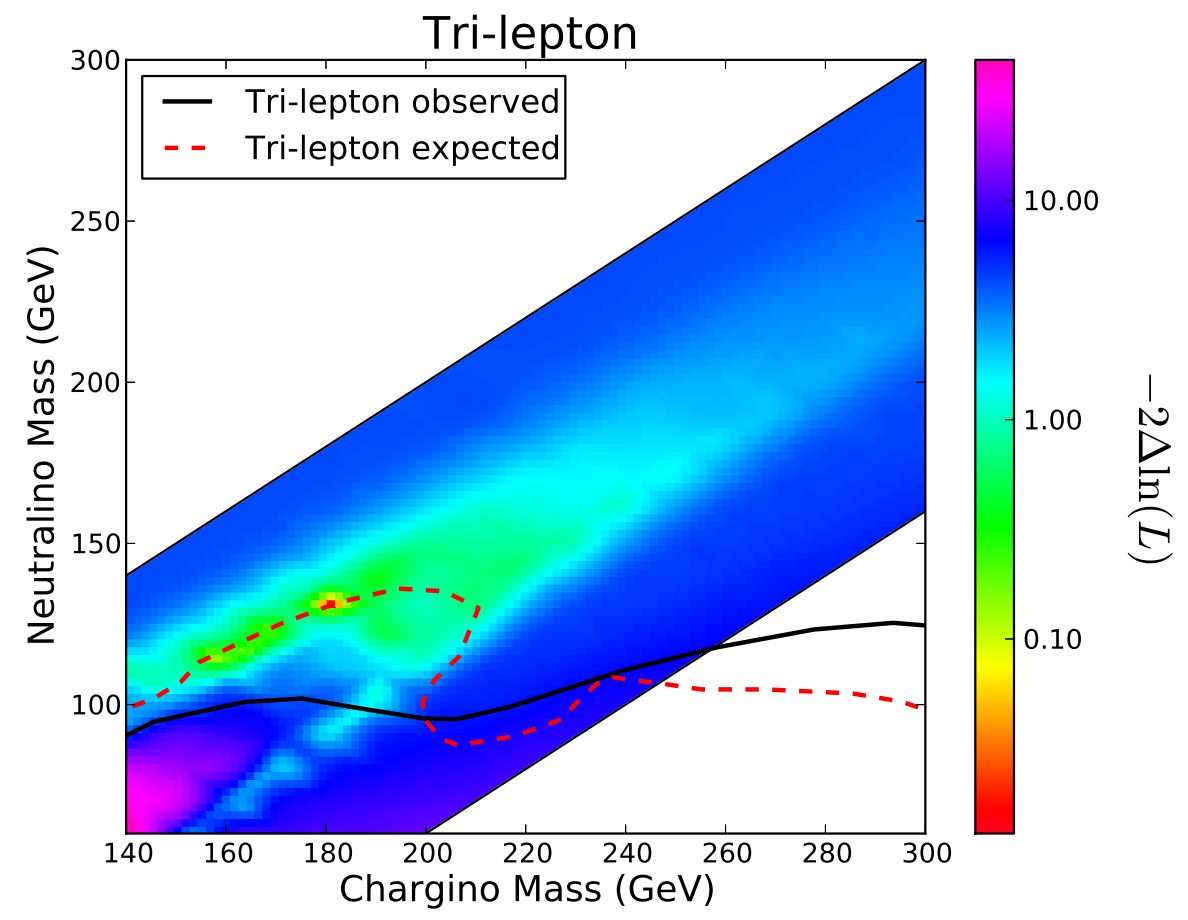

Figure 2. The distribution of $-2 \ln L$ as a function of the masses of the lightest chargino, $m_{\tilde{\chi}_{1}^{ \pm}}$, and lightest neutralino, $m_{\tilde{\chi}_{1}^{0}}$. All signal regions in the ATLAS tri-lepton search for charginos and neutralinos are used in the fit [24]. Also shown are the observed and expected 95\% CLs exclusion lines for the same search.

in eq. (4.2) and (4.3). We scan over the $\tilde{\chi}_{1}^{ \pm}=\tilde{\chi}_{2}^{0}$ and $\tilde{\chi}_{1}^{0}$ mass space and remind readers that we consider $\tilde{\chi}_{1}^{ \pm}, \tilde{\chi}_{2}^{0}$ to be purely wino and $\tilde{\chi}_{1}^{0}$ bino.

The best fit point for our model is to be found with the following parameters,

$$
\begin{aligned}
m_{\tilde{\chi}_{1}^{ \pm}}=m_{\tilde{\chi}_{2}^{0}} & =180_{-50}^{+90} \mathrm{GeV}, \\
m_{\tilde{\chi}_{1}^{0}} & =130_{-30}^{+60} \mathrm{GeV} .
\end{aligned}
$$

Compared to the SM, including $\tilde{\chi}_{1}^{ \pm}, \tilde{\chi}_{2}^{0}$ production reduces the log-likelihood of the fit by 4.3 which corresponds to 1.6- $\sigma$ once the extra degrees of freedom in the fit are considered.

As can be seen in figure 2, the best fit point lies well above the current experimental exclusion. However, one should notice that the expected exclusion goes almost exactly through our best fit point. Consequently, the current experimental analysis is sensitive to the model we propose. Indeed, if the excess present in the data is only due to a statistical fluctuation, one would expect the point to be ruled out in the forthcoming LHC run at $14 \mathrm{TeV}$. The breakdown of signal regions with significant contributions from our model point is given in table 3 of appendix A.

Finally we also note that at the best fit point our model has a mass difference $m_{\tilde{\chi}_{1}^{ \pm}, \tilde{\chi}_{2}^{0}}-$ $m_{\tilde{\chi}_{1}^{0}} \sim 50 \mathrm{GeV}$. Consequently, our model contributes negligibly to $W^{ \pm} Z^{0}$ measurements 


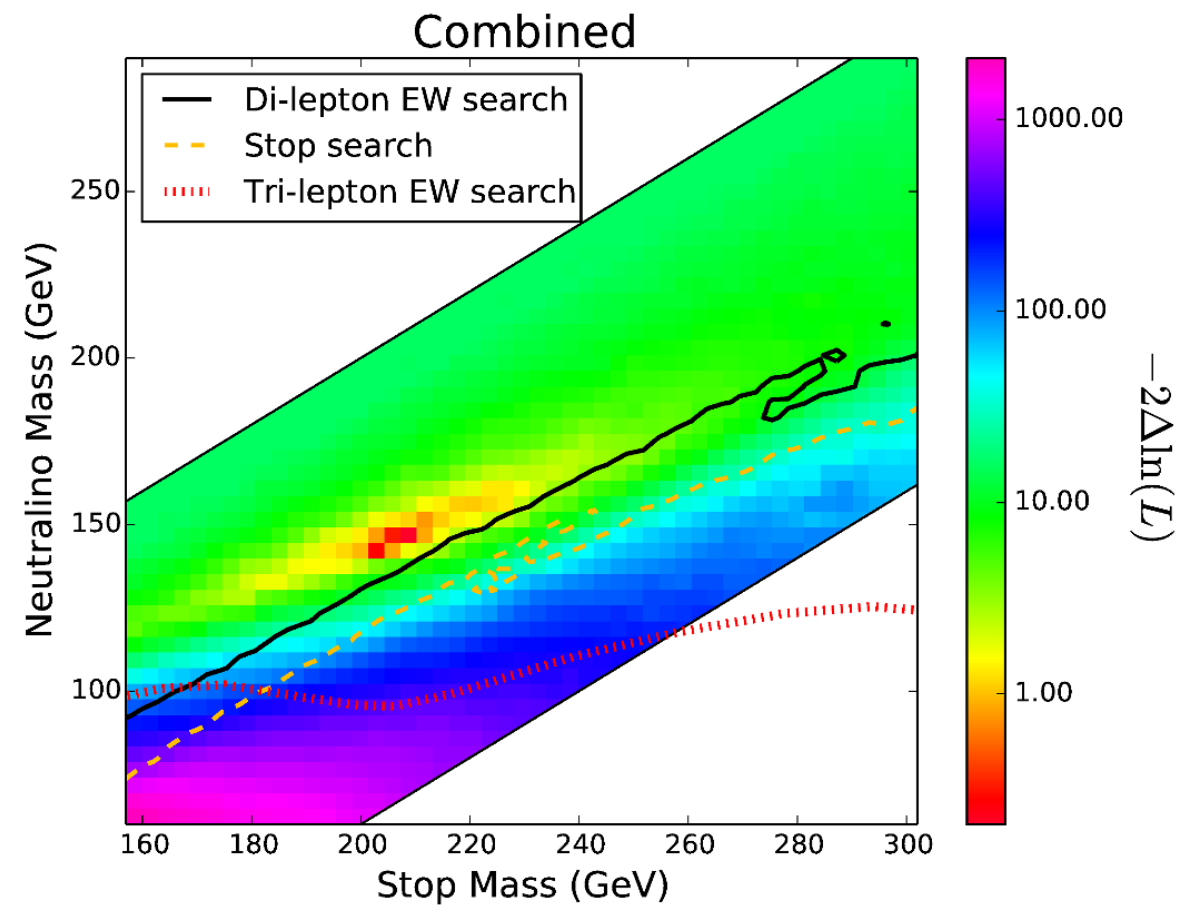

Figure 3. The distribution of $-2 \ln L$ as a function of the masses of the stop, $m_{\tilde{t}_{1}}$, and lightest neutralino, $m_{\tilde{\chi}_{1}^{0}}$. All of the signal regions given in the $W^{+} W^{-}$measurements, di-lepton and trilepton searches, table 1 are included in the fit. Also shown are the 95\% CLs exclusion lines given by the dedicated ATLAS di-lepton [25], tri-lepton [24] and stop [28] searches.

since the invariant mass of the leptons produced in the $\tilde{\chi}_{2}^{0}$ decay lies outside the normal mass window defined for the $Z^{0}$.

\subsection{Combined analysis}

One should already notice that the best-fit points for both $\tilde{t}_{1} \tilde{t}_{1}^{*}$ production and $\tilde{\chi}_{1}^{ \pm} \tilde{\chi}_{2}^{0}$ production lie very close and well within the $1-\sigma$ regions. We therefore perform a combined fit again as a function of $m_{\tilde{t}_{1}}$ and $m_{\tilde{\chi}_{1}^{0}}$ whilst keeping the mass splitting $m_{\tilde{t}_{1}}-m_{\tilde{\chi}_{1}^{ \pm}, \tilde{\chi}_{2}^{0}}=7 \mathrm{GeV}$.

The result is shown in figure 3 and we find the best fit point for our model to be,

$$
\begin{aligned}
m_{\tilde{t}_{1}} & =202_{-25}^{+35} \mathrm{GeV}, \\
m_{\tilde{\chi}_{1}^{0}} & =140_{-15}^{+25} \mathrm{GeV} .
\end{aligned}
$$

Comparing with the SM we find a reduction in the log-likelihood of 15.4 which corresponds to 3.5- $\sigma$ once the extra degrees of freedom in the fit are considered. In table 2 we show the breakdown of the different signal regions that display significant excesses and the improvement in the standard deviation due to our model. We see that all the $W^{+} W^{-}$measurements (including the Higgs) present a compelling improvement in the agreement with data.

The di-lepton measurements dominate the fit and thus the improvement in the trilepton signal regions is less stark. However, we still improve the compatibility with data 


\begin{tabular}{|llrrrrr|}
\hline Study & SR & Obs & Exp & $\begin{array}{r}\text { SM } \\
\text { s.d. }\end{array}$ & $\begin{array}{r}\text { Best } \\
\text { fit exp }\end{array}$ & $\begin{array}{r}\text { Best } \\
\text { fit s.d }\end{array}$ \\
\hline Atlas $W^{+} W^{-}(7 \mathrm{TeV})[5]$ & Combined & 1325 & $1219 \pm 87$ & $1.1-\sigma$ & 119 & $0.1-\sigma$ \\
\hline CMS $W^{+} W^{-}(7 \mathrm{TeV})[7]$ & Combined & 1134 & $1076 \pm 62$ & $0.8-\sigma$ & 89 & $0.4-\sigma$ \\
\hline CMS $W^{+} W^{-}(8 \mathrm{TeV})[6]$ & Combined & 1111 & $986 \pm 60$ & $1.8-\sigma$ & 83 & $0.6-\sigma$ \\
\hline ATLAS Higgs & $W W$ CR & 3297 & $3110 \pm 186$ & $0.9-\sigma$ & 374 & $0.9-\sigma$ \\
{$[27]$} & Higgs SR & 3615 & $3288 \pm 220$ & $1.4-\sigma$ & 501 & $0.6-\sigma$ \\
\hline AtLAS $\tilde{q}$ and $\tilde{g}$ & Di-muon & 7 & $1.7 \pm 1$ & $2.5-\sigma$ & 2.7 & $1.2-\sigma$ \\
$(1-2 \ell)[23]$ & & & & & & \\
\hline ATLAS Electroweak & SR0 $\tau$ a01 & 36 & $23 \pm 4$ & $2.1-\sigma$ & 2.8 & $1.6-\sigma$ \\
$(3 \ell)[24]$ & SR0 $\tau \mathrm{a} 06$ & 13 & $6.6 \pm 1.9$ & $1.9-\sigma$ & 1.5 & $1.4-\sigma$ \\
\hline
\end{tabular}

Table 2. Significant excesses present in the dataset under investigation. We give the signal region (SR) of interest, the observed number of events (Obs), the expected number of events (Exp) along with the associated systematic error. The SM standard deviation (s.d.) for each signal region is also given along with the expected number of events for our model best fit and the associated standard deviation. We only show the systematic error on the signal regions but the statistical errors are also included in the fit.

including a reduction in the standard deviation from 2.1- $\sigma \rightarrow 1.6-\sigma$ for the signal region 'SR0 $\tau \mathrm{a} 01$ '.

Another question one may ask is how does the fit quality change as the mass splitting between the stop and chargino is increased? If we increase the mass splitting to $15 \mathrm{GeV}$ (compared to the $7 \mathrm{GeV}$ presented), we find that the best fit point still has $m_{\tilde{t_{1}}}=202 \mathrm{GeV}$. However, the minimum of the log-likelihood increases by $\sim 1$, since the direct stop searches now become more sensitive to the model. This is due to the $b$-quark in the $\tilde{t}_{1} \rightarrow b \tilde{\chi}_{1}^{ \pm}$decay being harder and thus easier to reconstruct. As the mass difference is increased more, we expect the fit quality to deteriorate further.

\section{Discussion}

If the observed excess in $W^{+} W^{-}$measurements is confirmed with a larger significance in the full $8 \mathrm{TeV}$ dataset, it will be important to confirm its BSM origin. As argued in ref. [2] a useful observable [55-57] for that purpose is

$$
\cos \theta_{\ell \ell}^{*}=\tanh \left(\frac{\Delta \eta_{\ell \ell}}{2}\right), \quad \Delta \eta_{\ell \ell}=\eta_{\ell_{1}}-\eta_{\ell_{2}},
$$

where $\Delta \eta_{\ell \ell}$ is a difference of the pseudo-rapidities between the leading and the trailing lepton. This variable is a cosine of the polar angle of the leptons with respect to the beam axis in the frame where the pseudo-rapidities of the leptons are equal and opposite, as discussed in ref. [55]. Being a function of the difference of pseudo-rapidities, it is longitudinally 


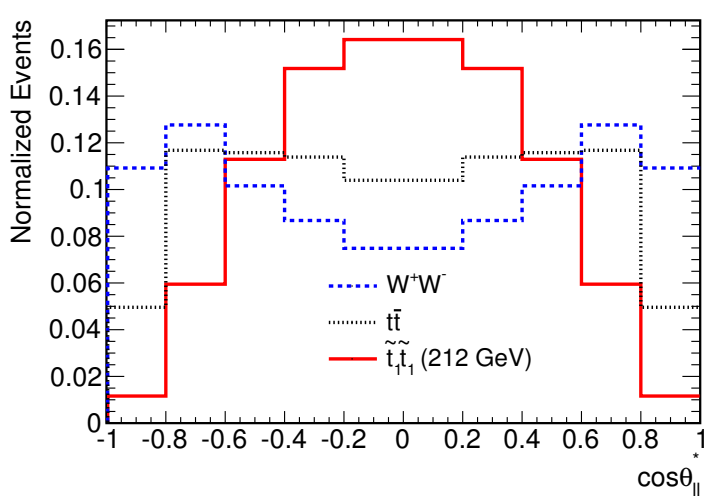

(a)

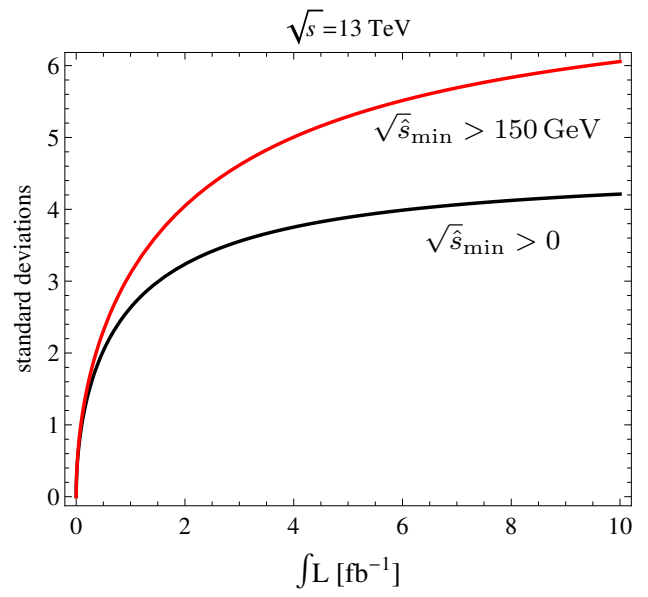

(b)

Figure 4. (a) The distribution of $\cos \theta_{\ell \ell}^{*}$ for leptons produced from SM $W^{+} W^{-}$and $t \bar{t}$ events, and from $\tilde{t}_{1} \tilde{t}_{1}^{*}$ events for our best fit point. (b) The significance of distinguishing the SM-only and $\mathrm{SM}+\tilde{t}_{1} \tilde{t}_{1}^{*}$ case as a function of an integrated luminosity using the asymmetry eq. (5.3). The red curve shows the significance with the cut eq. (5.2), while the black curve without the $\sqrt{\hat{s}}_{\text {min }}$ cut.

boost-invariant. Following a discussion in ref. [2] we extend its sensitivity by requiring

$$
\sqrt{\hat{s}}_{\text {min }}=\sqrt{E^{2}-P_{z}^{2}}+E_{T}^{\text {miss }}>150 \mathrm{GeV}
$$

with $E, P_{z}$ being the total energy and longitudinal momentum of the reconstructed leptons. The resulting distribution is shown in figure 4(a). In figure 4(b) we show the evolution of the significance as a function of luminosity collected at $13 \mathrm{TeV}$ for the asymmetry defined as

$$
\mathcal{A}=\frac{N\left(\left|\cos \theta_{\ell \ell}^{*}\right|>0.5\right)-N\left(\left|\cos \theta_{\ell \ell}^{*}\right|<0.5\right)}{N_{\text {tot }}}
$$

where $N(\ldots)$ is the number of events fulfilling the respective condition. The value of the asymmetry for stop signal alone is $\mathcal{A}_{\tilde{t}_{1}}=-0.52$, for the $\mathrm{SM}$ contribution $\mathcal{A}_{\mathrm{SM}}=0.12$ and for the sum of stop and SM contributions $\mathcal{A}_{\mathrm{SM}+\tilde{t}_{1}}=-0.04$. After $\sim 5 \mathrm{fb}^{-1}$ a $5-\sigma$ discrimination between SUSY and the SM should be achievable while with the full $8 \mathrm{TeV}$ data sample the expected significance is $3-\sigma$. In addition a shape analysis of the $\cos \theta_{\ell \ell}^{*}$ distribution can further enhance significance of the discovery [2].

Soft $b$-jets coming from stop decays could also be exploited to distinguish the stop contribution from genuine $W^{+} W^{-}$production. A detailed analysis of $t \bar{t}$ background would be required in this case. This technique would require a detailed detector simulation and is beyond the scope of this study. We note however, that observation of such a signal would clearly point to the BSM partners of the third generation quarks. Another possible method is to study correlations of tagging jets [58] that can be different between stop production and background processes.

With a higher integrated luminosity and production cross section when the LHC moves to $13 \mathrm{TeV}$ one could envisage a study of the di-lepton distribution from the neutralino 
$\tilde{\chi}_{2}^{0}$ decays. This would enable a direct measurement of the mass difference, $m_{\tilde{\chi}_{2}^{0}}-m_{\tilde{\chi}_{1}^{0}}$. Together with the cross section measurement a more precise measurement of absolute masses could be expected.

A final enticing prospect is the hope that other SUSY states could be in reach of the LHC. The naturalness arguments that predict light stops also dictate that the gluino should not be much heavier than the current bounds. Perhaps with the extended centre of mass energy, these states will become fully accessible. Related to the question of naturalness is the mass of the higgsinos. In our model we chose the bino to be lightest SUSY particle and the wino to be close in mass to the stop. However, we believe either of these states could be replaced by higgsinos and the fit would proceed with a similar result. Another possibility is that the higgsinos could lie just above the stop mass and in this case they would have no effect on the phenomenology presented here.

In this study we chose what we think is one of the simplest SUSY model in order to explain the current LHC excesses by only varying the stop and LSP mass. In doing so, we took the 'canonical' choice of a bino LSP and wino NLSP and a primarily righthanded stop to avoid any possible constraints from Higgs data or electroweak precision tests $[59,60]$. We anticipate that this simple choice may attract criticism since it will not predict the correct dark matter relic density. However, we believe that there are two trivial solutions to this problem that will only minimally alter the LHC phenomenology. Firstly, by allowing a generic mixing of the parameters of the neutralino and chargino mass matrix, a 'well tempered' LSP [61] can be achieved. Secondly, introducing an extra particle that lies close in mass to the LSP (for example a NMSSM singlino [62] or a right-handed slepton) can lead to effective co-annihilation [63, 64].

In addition, one can also question whether the outstanding problem of the anomalous magnetic moment of the muon could be addressed. Essentially, we would require light sleptons in the vicinity of the current best-fit point. Simply by placing their mass above the chargino they would clearly have a non-negligible impact on the branching ratios of chargino. One of the consequences could be shifting the preferred chargino mass higher since with the enhanced leptonic branching ratio the lower cross section would suffice reproduce the current excess. If lighter, they could also play a role in enhancing annihilation cross section of the LSP and also decays of charginos via an intermediate slepton.

The reason we chose not to include these effects is that in the general MSSM, the parameter space allows a huge range of possibilities that the current data cannot distinguish. We worried that adding (at least two) additional parameters to fit two measurements may detract and distract from the fact that the current LHC data can be fit with a far simpler model.

One should also mention of course that on a phenomenological level, there is no reason to conclude that a SUSY model fits the current data best and it is likely that many other models will also be able match satisfactorily.

\section{Conclusions}

In this study we have analysed a simplified model with light stop quarks, charginos and neutralinos. A detailed scan of the available parameter space was performed by checking 
the compatibility of this model with a number of searches and measurements performed by the LHC experiments. The most significant studies in our analysis are the $W^{+} W^{-}$cross section measurements, Higgs spin measurement and SUSY tri-lepton searches. All of them show moderate excesses in the observed number of events.

We found that the inclusion of stop production improves the fit to the data with respect to the SM with a reduction in the log-likelihood of 15.5. Once the extra degrees of freedom in the fit are considered, this corresponds to 3.5- $\sigma$ difference. The parameter point that best fits the available data is given by $m_{\tilde{t}_{1}}=202 \mathrm{GeV}, m_{\tilde{\chi}_{1}^{ \pm}}=m_{\tilde{\chi}_{2}^{0}}=195 \mathrm{GeV}$, and $m_{\tilde{\chi}_{1}^{0}}=140 \mathrm{GeV}$. The production of light stops, $p p \rightarrow \tilde{t}_{1} \tilde{t}_{1}^{*}$, contributes to di-lepton observables, while chargino-neutralino production, $p p \rightarrow \tilde{\chi}_{1}^{ \pm} \tilde{\chi}_{2}^{0}$ helps explain the excess in the SUSY tri-lepton search.

An additional observable based on the rapidities of the final state leptons could further increase the significance of the discovery. It provides a strong hint about the BSM nature of the excess in $W^{+} W^{-}$cross section measurements.

While the available measurements are not conclusive as whether new physics has indeed been observed at the current LHC run, the several experimental hints presented here point to an exciting possibility that requires further investigation. It is worth noting that several different studies point to the same region in parameter space that is also not excluded by the other available searches.

The situation may become more clear when the final measurement of $W^{+} W^{-}$production cross section at $\sqrt{s}=8 \mathrm{TeV}$ is released by Atlas and CMS. However, the final answer will be provided by the future measurements with the LHC at $13 \mathrm{TeV}$. With a higher centre-of-mass energy and an integrated luminosity increased by an order of magnitude, the nature of the excesses, if they persist, can be studied in detail.

Note. Simultaneous to our work, ref. [65] have also investigated the $W^{+} W^{-}$excess with SUSY models and results similar to the ones presented here.

\section{Acknowledgments}

We would like to thank the crucial help of Daniel Schmeier in developing Checkmate and helping with many of the analyses used here. We would also like to thank Matthew Dolan for pointing out errors in the way we had included Higgs events in the $W^{+} W^{-}$measurements. This work has been partially supported by the MICINN, Spain, under contract FPA2010-17747; Consolider-Ingenio CPAN CSD2007-00042; and the European Commission under contract PITN-GA-2009-237920. JSK also thanks the Spanish MINECO Centro de excelencia Severo Ochoa Program under grant SEV-2012-0249. The work of K.S. was supported in part by the London Centre for Terauniverse Studies (LCTS), using funding from the European Research Council via the Advanced Investigator Grant 267352.

\section{A Signal region events}




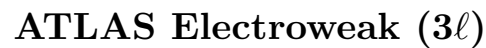

\begin{tabular}{|lccccc|}
\hline $\begin{array}{l}\text { Signal } \\
\text { Region }\end{array}$ & $\begin{array}{c}\text { Observed } \\
\text { events }\end{array}$ & $\begin{array}{c}\text { Expected } \\
\text { events }\end{array}$ & $\begin{array}{c}\text { Standard Model } \\
\text { standard deviation }\end{array}$ & $\begin{array}{c}\text { Best fit } \\
\text { events }\end{array}$ & $\begin{array}{c}\text { Best fit } \\
\text { standard deviation }\end{array}$ \\
\hline SR0 $\tau \mathrm{a} 01$ & 36 & $23 \pm 4$ & $2.1-\sigma$ & 2.8 & $1.6-\sigma$ \\
SR0 $\tau \mathrm{a} 02$ & 5 & $4.2 \pm 1.5$ & $0.8-\sigma$ & 1.1 & $0.6-\sigma$ \\
SR0 $\tau \mathrm{a} 03$ & 9 & $10.6 \pm 1.8$ & $0.7-\sigma$ & 0.6 & $0.8-\sigma$ \\
SR0 $\tau \mathrm{a} 04$ & 9 & $8.5 \pm 1.7$ & $0.6-\sigma$ & 0.7 & $0.5-\sigma$ \\
SR0 $\tau \mathrm{a} 05$ & 11 & $12.9 \pm 2.4$ & $0.7-\sigma$ & 1.4 & $0.9-\sigma$ \\
$\mathrm{SR} 0 \tau \mathrm{a} 06$ & 13 & $6.6 \pm 1.9$ & $1.9-\sigma$ & 1.5 & $1.4-\sigma$ \\
$\mathrm{SR} 0 \tau \mathrm{a} 07$ & 15 & $14.1 \pm 2.2$ & $0.6-\sigma$ & 0.6 & $0.5-\sigma$ \\
$\mathrm{SR} 0 \tau \mathrm{a} 08$ & 1 & $1.1 \pm 0.4$ & $0.4-\sigma$ & 0.2 & $0.4-\sigma$ \\
$\mathrm{SR} 0 \tau \mathrm{a} 09$ & 28 & $22.4 \pm 3.6$ & $1.2-\sigma$ & 0.2 & $1.1-\sigma$ \\
$\mathrm{SR} 0 \tau \mathrm{a} 10$ & 24 & $16.4 \pm 2.4$ & $1.6-\sigma$ & 0.4 & $1.5-\sigma$ \\
$\mathrm{SR} 0 \tau \mathrm{a} 11$ & 29 & $27 \pm 5$ & $0.8-\sigma$ & 0.4 & $0.8-\sigma$ \\
$\mathrm{SR} 0 \tau \mathrm{a} 12$ & 8 & $5.5 \pm 1.5$ & $1.1-\sigma$ & 0.3 & $1.0-\sigma$ \\
\hline
\end{tabular}

Table 3. Signal regions from the ATLAS electroweak $3 \ell$ search [24] where the SUSY signal contributes to the fit. We give the signal region of interest, the observed number of events, the expected number of events along with the associated systematic error. The SM standard deviation for each signal region is also given along with the expected number of events for our model best fit and the associated standard deviation. We only show the systematic error on the signal regions but the statistical errors are also included in the fit.

Open Access. This article is distributed under the terms of the Creative Commons Attribution License (CC-BY 4.0), which permits any use, distribution and reproduction in any medium, provided the original author(s) and source are credited.

\section{References}

[1] S.P. Martin, A supersymmetry primer, Adv. Ser. Direct. High Energy Phys. 21 (2010) 1 [hep-ph/9709356] [inSPIRE].

[2] K. Rolbiecki and K. Sakurai, Light stops emerging in WW cross section measurements?, JHEP 09 (2013) 004 [arXiv: 1303.5696] [INSPIRE].

[3] I.-W. Kim, M. Papucci, K. Sakurai and A. Weiler, ATOM: Automated Testing Of Models, to appear.

[4] M. Drees, H. Dreiner, D. Schmeier, J. Tattersall and J.S. Kim, CheckMATE: confronting your favourite new physics model with LHC data, arXiv:1312.2591 [INSPIRE].

[5] ATLAS collaboration, Measurement of $W^{+} W^{-}$production in pp collisions at $\sqrt{s}=7 \mathrm{TeV}$ with the ATLAS detector and limits on anomalous $W W Z$ and $W W \gamma$ couplings, Phys. Rev. D 87 (2013) 112001 [arXiv:1210.2979] [INSPIRE]. 
[6] CMS collaboration, Measurement of $W^{+} W^{-}$and $Z Z$ production cross sections in $p p$ collisions at $\sqrt{s}=8 \mathrm{TeV}$, Phys. Lett. B 721 (2013) 190 [arXiv:1301.4698] [InSPIRE].

[7] CMS collaboration, Measurement of the $W^{+} W^{-}$cross section in pp collisions at $\sqrt{s}=7$ TeV and limits on anomalous $W W \gamma$ and $W W Z$ couplings, Eur. Phys. J. C 73 (2013) 2610 [arXiv: 1306.1126] [InSPIRE].

[8] J. Baglio, L.D. Ninh and M.M. Weber, Massive gauge boson pair production at the LHC: a next-to-leading order story, Phys. Rev. D 88 (2013) 113005 [arXiv:1307.4331] [INSPIRE].

[9] S. Dawson, I.M. Lewis and M. Zeng, Threshold resummed and approximate next-to-next-to-leading order results for $W^{+} W^{-}$pair production at the LHC, Phys. Rev. D 88 (2013) 054028 [arXiv: 1307.3249] [INSPIRE].

[10] Herafitter developers' team collaboration, P. Belov et al., Parton distribution functions at LO, NLO and NNLO with correlated uncertainties between orders, Eur. Phys. J. C 74 (2014) 3039 [arXiv: 1404.4234] [InSPIRE].

[11] D. Curtin, P. Jaiswal and P. Meade, Charginos hiding in plain sight, Phys. Rev. D 87 (2013) 031701 [arXiv: 1206.6888 ] [INSPIRE].

[12] D. Curtin, P. Jaiswal, P. Meade and P.-J. Tien, Casting light on BSM physics with SM standard candles, JHEP 08 (2013) 068 [arXiv:1304.7011] [INSPIRE].

[13] ATLAS collaboration, Measurement of the inclusive $W^{ \pm}$and $Z / \gamma$ cross sections in the electron and muon decay channels in pp collisions at $\sqrt{s}=7 \mathrm{TeV}$ with the ATLAS detector, Phys. Rev. D 85 (2012) 072004 [arXiv:1109.5141] [INSPIRE].

[14] CMS collaboration, Measurement of inclusive $W$ and $Z$ boson production cross sections in $p p$ collisions at $\sqrt{s}=8 \mathrm{TeV}$, Phys. Rev. Lett. 112 (2014) 191802 [arXiv:1402.0923] [INSPIRE].

[15] CMS collaboration, Measurement of the differential and double-differential Drell-Yan cross sections in proton-proton collisions at $\sqrt{s}=7$ TeV, JHEP 12 (2013) 030 [arXiv:1310.7291] [INSPIRE].

[16] ATLAS collaboration, Measurement of the high-mass Drell-Yan differential cross-section in pp collisions at $\sqrt{s}=7 \mathrm{TeV}$ with the ATLAS detector, Phys. Lett. B 725 (2013) 223 [arXiv: 1305.4192] [INSPIRE].

[17] ATLAS collaboration, Measurement of $W Z$ production in proton-proton collisions at $\sqrt{s}=7 \mathrm{TeV}$ with the ATLAS detector, Eur. Phys. J. C 72 (2012) 2173 [arXiv:1208.1390] [INSPIRE].

[18] ATLAS collaboration, $A$ measurement of $W Z$ production in proton-proton collisions at $\sqrt{s}=8 \mathrm{TeV}$ with the ATLAS detector, ATLAS-CONF-2013-021, CERN, Geneva Switzerland (2013).

[19] CMS collaboration, Measurement of WZ production rate, CMS-PAS-SMP-12-006, CERN, Geneva Switzerland (2013).

[20] ATLAS collaboration, Measurement of $Z Z$ production in pp collisions at $\sqrt{s}=7 \mathrm{TeV}$ and limits on anomalous $Z Z Z$ and $Z Z \gamma$ couplings with the ATLAS detector, JHEP 03 (2013) 128 [arXiv:1211.6096] [INSPIRE].

[21] ATLAS collaboration, Measurement of the total $Z Z$ production cross section in proton-proton collisions at $\sqrt{s}=8 \mathrm{TeV}$ in $20 \mathrm{fb}^{-1}$ with the ATLAS detector, ATLAS-CONF-2013-020, CERN, Geneva Switzerland (2013). 
[22] CMS collaboration, Measurement of the $Z Z$ production cross section and anomalous trilinear gauge couplings in $\ell \ell \ell^{\prime} \ell^{\prime}$ decays at $\sqrt{s}=8$ TeV at the LHC, CMS-PAS-SMP-13-005, CERN, Geneva Switzerland (2013).

[23] ATLAS collaboration, Search for squarks and gluinos in events with isolated leptons, jets and missing transverse momentum at $\sqrt{s}=8 \mathrm{TeV}$ with the ATLAS detector, ATLAS-CONF-2013-062, CERN, Geneva Switzerland (2013).

[24] ATLAS collaboration, Search for direct production of charginos and neutralinos in events with three leptons and missing transverse momentum in $\sqrt{s}=8$ TeV pp collisions with the ATLAS detector, JHEP 04 (2014) 169 [arXiv:1402.7029] [INSPIRE].

[25] ATLAS collaboration, Search for direct production of charginos, neutralinos and sleptons in final states with two leptons and missing transverse momentum in pp collisions at $\sqrt{s}=8 \mathrm{TeV}$ with the ATLAS detector, JHEP 05 (2014) 071 [arXiv:1403.5294] [INSPIRE].

[26] ATLAS collaboration, Measurements of the properties of the Higgs-like boson in the $W W^{(*)} \rightarrow \ell \nu \ell \nu$ decay channel with the ATLAS detector using $25 \mathrm{fb}^{-1}$ of proton-proton collision data, ATLAS-CONF-2013-030, CERN, Geneva Switzerland (2013).

[27] ATLAS collaboration, Study of the spin properties of the Higgs-like particle in the

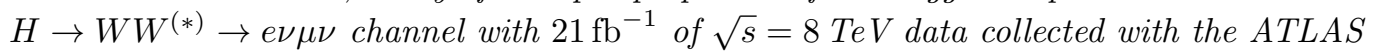
detector, ATLAS-CONF-2013-031, CERN, Geneva Switzerland (2013).

[28] ATLAS collaboration, Search for direct top-squark pair production in final states with two leptons in pp collisions at $\sqrt{s}=8$ TeV with the ATLAS detector, JHEP 06 (2014) 124 [arXiv: 1403.4853] [INSPIRE].

[29] ATLAS collaboration, Search for direct top squark pair production in final states with one isolated lepton, jets and missing transverse momentum in $\sqrt{s}=8$ TeV pp collisions using $21 \mathrm{fb}^{-1}$ of ATLAS data, ATLAS-CONF-2013-037, CERN, Geneva Switzerland (2013).

[30] J.E. Camargo-Molina, B. Garbrecht, B. O'Leary, W. Porod and F. Staub, Constraining the natural MSSM through tunneling to color-breaking vacua at zero and non-zero temperature, Phys. Lett. B 737 (2014) 156 [arXiv:1405.7376] [INSPIRE].

[31] A. Buckley et al., Rivet user manual, Comput. Phys. Commun. 184 (2013) 2803 [arXiv: 1003.0694] [INSPIRE].

[32] DELPHES 3 collaboration, J. de Favereau et al., DELPHES 3, a modular framework for fast simulation of a generic collider experiment, JHEP 02 (2014) 057 [arXiv:1307.6346] [INSPIRE].

[33] M. Cacciari, G.P. Salam and G. Soyez, FastJet user manual, Eur. Phys. J. C 72 (2012) 1896 [arXiv:1111.6097] [INSPIRE].

[34] M. Cacciari and G.P. Salam, Dispelling the $N^{3}$ myth for the $k_{t}$ jet-finder, Phys. Lett. B 641 (2006) 57 [hep-ph/0512210] [INSPIRE].

[35] M. Cacciari, G.P. Salam and G. Soyez, The anti- $k_{t}$ jet clustering algorithm, JHEP 04 (2008) 063 [arXiv:0802.1189] [INSPIRE].

[36] C.G. Lester and D.J. Summers, Measuring masses of semiinvisibly decaying particles pair produced at hadron colliders, Phys. Lett. B 463 (1999) 99 [hep-ph/9906349] [INSPIRE].

[37] A. Barr, C. Lester and P. Stephens, $m_{T 2}$ : the truth behind the glamour, J. Phys. G 29 (2003) 2343 [hep-ph/0304226] [INSPIRE]. 
[38] H.-C. Cheng and Z. Han, Minimal kinematic constraints and $m_{T 2}$, JHEP 12 (2008) 063 [arXiv:0810.5178] [INSPIRE].

[39] M. Bahr et al., HERWIG++ physics and manual, Eur. Phys. J. C 58 (2008) 639 [arXiv: 0803.0883] [INSPIRE].

[40] J. Bellm et al., HERWIG++ 2.7 release note, arXiv:1310.6877 [INSPIRE].

[41] W. Beenakker, R. Hopker, M. Spira and P.M. Zerwas, Squark and gluino production at hadron colliders, Nucl. Phys. B 492 (1997) 51 [hep-ph/9610490] [INSPIRE].

[42] W. Beenakker, M. Krämer, T. Plehn, M. Spira and P.M. Zerwas, Stop production at hadron colliders, Nucl. Phys. B 515 (1998) 3 [hep-ph/9710451] [INSPIRE].

[43] W. Beenakker et al., Soft-gluon resummation for squark and gluino hadroproduction, JHEP 12 (2009) 041 [arXiv: 0909.4418] [INSPIRE].

[44] W. Beenakker et al., Supersymmetric top and bottom squark production at hadron colliders, JHEP 08 (2010) 098 [arXiv: 1006.4771] [INSPIRE].

[45] W. Beenakker et al., The production of charginos/neutralinos and sleptons at hadron colliders, Phys. Rev. Lett. 83 (1999) 3780 [Erratum ibid. 100 (2008) 029901] [hep-ph/9906298] [INSPIRE].

[46] P.M. Nadolsky et al., Implications of CTEQ global analysis for collider observables, Phys. Rev. D 78 (2008) 013004 [arXiv: 0802.0007] [INSPIRE].

[47] H. Dreiner, M. Krämer and J. Tattersall, Exploring QCD uncertainties when setting limits on compressed supersymmetric spectra, Phys. Rev. D 87 (2013) 035006 [arXiv:1211.4981] [INSPIRE].

[48] H.K. Dreiner, M. Krämer and J. Tattersall, How low can SUSY go? Matching, monojets and compressed spectra, Europhys. Lett. 99 (2012) 61001 [arXiv:1207.1613] [INSPIRE].

[49] CMS collaboration, Searches for electroweak production of charginos, neutralinos and sleptons decaying to leptons and $W, Z$ and Higgs bosons in pp collisions at $8 \mathrm{TeV}$, Eur. Phys. J. C 74 (2014) 3036 [arXiv:1405.7570] [INSPIRE].

[50] CMS collaboration, Search for top-squark pair production in the single-lepton final state in pp collisions at $\sqrt{s}=8 \mathrm{TeV}$, Eur. Phys. J. C 73 (2013) 2677 [arXiv:1308.1586] [InSPIRE].

[51] CMS collaboration, Search for new physics in events with same-sign dileptons and $b$ jets in pp collisions at $\sqrt{s}=8 \mathrm{TeV}$, JHEP 03 (2013) 037 [Erratum ibid. 07 (2013) 041] [arXiv: 1212.6194] [INSPIRE].

[52] CMS collaboration, Search for electroweak production of charginos, neutralinos and sleptons using leptonic final states in pp collisions at $8 \mathrm{TeV}$, CMS-PAS-SUS-13-006, CERN, Geneva Switzerland (2013).

[53] ATLAS collaboration, Search for strongly produced supersymmetric particles in decays with two leptons at $\sqrt{s}=8 \mathrm{TeV}$, ATLAS-CONF-2013-089, CERN, Geneva Switzerland (2013).

[54] J. Conway, Calculation of cross section upper limits combining channels incorporating correlated and uncorrelated systematic uncertainties, CDF/PUB/STATISTICS/PUBLIC/6428, Fermilab Batavia U.S.A. (2005).

[55] A.J. Barr, Measuring slepton spin at the LHC, JHEP 02 (2006) 042 [hep-ph/0511115] [INSPIRE]. 
[56] R. Diener, S. Godfrey and T.A.W. Martin, Using final state pseudorapidities to improve s-channel resonance observables at the LHC, Phys. Rev. D 80 (2009) 075014 [arXiv:0909.2022] [INSPIRE].

[57] G. Moortgat-Pick, K. Rolbiecki and J. Tattersall, Early spin determination at the LHC?, Phys. Lett. B 699 (2011) 158 [arXiv:1102.0293] [InSPIRE].

[58] M.R. Buckley, T. Plehn and M.J. Ramsey-Musolf, Stop on top, Phys. Rev. D 90 (2014) 014046 [arXiv: 1403.2726] [INSPIRE].

[59] M. Drees and K. Hagiwara, Supersymmetric contribution to the electroweak $\rho$ parameter, Phys. Rev. D 42 (1990) 1709 [INSPIRE].

[60] S. Heinemeyer, W. Hollik, G. Weiglein and L. Zeune, Implications of LHC search results on the $W$ boson mass prediction in the MSSM, JHEP 12 (2013) 084 [arXiv:1311.1663] [INSPIRE].

[61] N. Arkani-Hamed, A. Delgado and G.F. Giudice, The well-tempered neutralino, Nucl. Phys. B 741 (2006) 108 [hep-ph/0601041] [INSPIRE].

[62] V. Barger, P. Langacker and H.-S. Lee, Lightest neutralino in extensions of the MSSM, Phys. Lett. B 630 (2005) 85 [hep-ph/0508027] [INSPIRE].

[63] S. Kraml, A.R. Raklev and M.J. White, NMSSM in disguise: discovering singlino dark matter with soft leptons at the LHC, Phys. Lett. B 672 (2009) 361 [arXiv:0811.0011] [INSPIRE].

[64] J.S. Kim and T.S. Ray, The Higgsino-Singlino world, arXiv:1405.3700 [InSPIRE].

[65] D. Curtin, P. Meade and P.-J. Tien, Natural SUSY in plain sight, arXiv:1406.0848 [INSPIRE]. 